\title{
Factores pronósticos y nuevos aspectos de la biología molecular en el cáncer de estómago resecable
}

\author{
F. J. Vizoso Piñeiro*,**, M. D. Corte Torres**, J. L. García Muñiz ${ }^{* * *}$
}

\section{Introducción}

A pesar de su incidencia declinante a partir de la segunda mitad del siglo XX, el cáncer gástrico sigue representando la segunda causa de muerte por cáncer, tras la ocasionada por el de pulmón'. Los factores pronósticos para este tumor se pueden clasificar en dos grandes grupos: los factores pronósticos convencionales y los biológicos. Dentro de los factores del primer grupo están los que dependen del paciente y del tumor, así como los relacionados con el tipo de tratamiento. Con respecto a este último tipo de factor, y considerando que el tratamiento más efectivo para este tipo de tumor es el quirúrgico, resulta absolutamente clave para lograr una resecabilidad completa del tumor (RO, de acuerdo a la UICC: no tumor residual macroscópico, ni microscópico tras la cirugía). Sin embargo, como se puede apreciar en la Figura 1, aun en los casos quirúrgicamente resecables, la probabilidad de supervivencia a los cinco años apenas supera el $40 \%$. Es más, la probabilidad de supervivencia libre de enfermedad es muy similar a la de supervivencia global, lo que da una idea de la agresividad biológica de estos tumores. Por ello, resulta importante en la práctica clínica, la consideración de factores pronósticos que nos permitan una evaluación más óptima de la agresividad de los carcinomas gástricos resecables quirúrgicamente, y que también posibiliten el establecimiento de nuevas dianas terapéuticas.

\section{Factores pronósticos clásicos}

Entre los factores pronósticos convencionales que han sido evaluados en el cáncer gástrico, se encuentran la edad y sexo de los pacientes, la localización tumoral, configuración macroscópica del tumor, estadio tumoral, tipo y grado

* Servicio de Cirugía General. Hospital de Jove. Gijón

** Instituto Universitario de Oncología del Principado de Asturias

*** Servicio de Cirugía General. Hospital General de Asturias. Oviedo

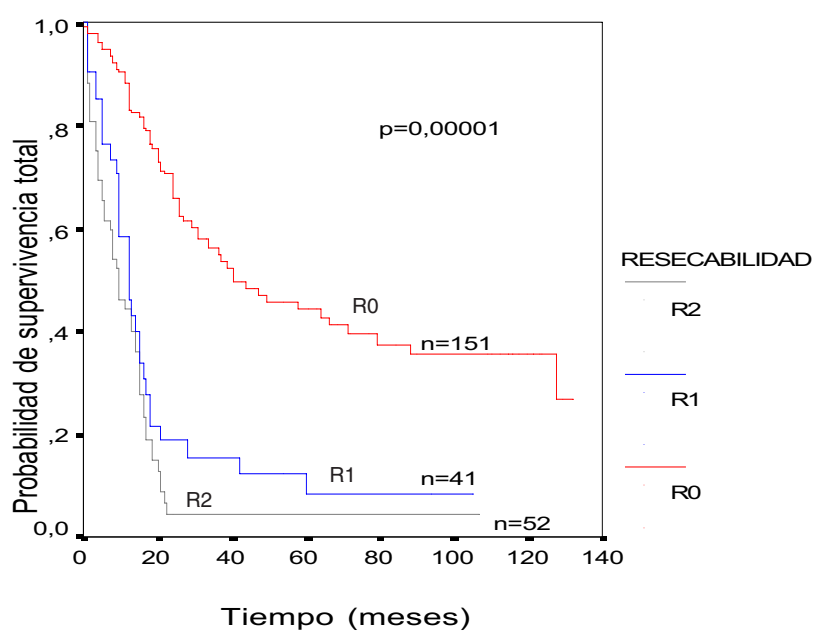

Fig. 1. Supervivencia total en función de la resecabilidad quirúrgica en 244 pacientes con cáncer gástrico (mediana de seguimiento: 28 meses).

histológico. El estadio tumoral, de acuerdo con la clasificación TNM, que es la más empleada en occidente, es el factor pronóstico más importante en los carcinomas gástricos resecables. La localización tumoral en cardias y en muñón gástrico de pacientes que fueron sometidos a gastrectomía parcial por enfermedad ulcerosa, también está asociada con un peor pronóstico. En general, la mayoría de los estudios indican que la configuración macroscópica del crecimiento de tipo linitis plástica (clasificación de Borrmann), el grado histológico indiferenciado, el tipo histológico difuso (criterios de Lauren), así como aspectos histopatológicos tales como la invasión vascular, perineural o linfática, se asocian con un peor pronóstico del cáncer gástrico resecable. De todos esos factores, en un estudio realizado sobre 151 pacientes con cáncer gástrico resecable que fueron sometidas a un período medio de seguimiento clínico de 36,6 meses (mediana: 24 meses) en los Hospitales Central de Asturias, de Oviedo y de Jove, de Gijón, tan sólo el estadio tumoral $(p=0,00001)$, la localización tumoral $(p=0,005)$ y el tipo histológico de los tumores $(p=0,004)$ estuvieron significativamente asociados con la supervivencia de los pacientes. 


\section{F. J. Vizoso Piñeiro y cols.}

\section{Factores pronósticos biológicos}

Uno de los mayores esfuerzos en la investigación oncológica actual, es tratar de trasladar nuevos aspectos biológicos del cáncer gástrico, de la investigación básica a la práctica clínica, con objeto de complementar la información aportada por los factores pronósticos clásicos y abrir la posibilidad de nuevas estrategias terapéuticas. En la última década se han ido investigando progresivamente en este tipo de neoplasia la mayoría de los parámetros de la biología molecular asociados al cáncer, tales como el contenido de ADN y los índices de proliferación celular, oncogenes y antioncogenes, factores de crecimiento y sus receptores, moléculas de adhesión, elementos de la matriz extracelular, enzimas proteolíticos y sus inhibidores, así como la expresión de determinados antígenos asociados a tumores.

\section{Contenido de ADN, índices de proliferación celular}

Se estima que en torno a un $50 \%$ de los carcinomas gástricos muestran un contenido anormal de $A D N$, así como índices elevados de proliferación celular, tales como porcentaje de células tumorales en fase $S$, y la expresión de antígenos de proliferación celular (ki67, p105, o actividad telomerasa. En general, la expresión elevada de esos parámetros se ha asociado con una mayor agresividad de los carcinomas gástricos y con un peor pronóstico de los pacientes ${ }^{2}$.

\section{Alteraciones genéticas}

Los carcinomas gástricos muestran frecuentemente mutaciones de oncogenes y de genes supresores tumorales. Las mejor caracterizadas son las del oncogén c-erbB-2 y la del gen supresor tumoral p53.

Existen una serie de evidencias clínicas y experimentales que apoyan el papel del c-erbB-2 en la patogénesis y agresividad clínica de los tumores malignos. Así, se ha observado que la introducción del oncogén en células normales causa transformación maligna, o que los ratones transgénicos que lo sobreexpresan desarrollan tumores. Se ha demostrado que un porcentaje significativo de carcinomas gástricos sobreexpresan el c-erbB-2. Además, nosotros hemos encontrado que las concentraciones intratumorales elevadas de la proteína ErbB-2 está asociada con una menor supervivencia total de los pacientes con cáncer gástrico, tanto irresecable ${ }^{3}$ como resecable quirúrgicamente ${ }^{4}$. Esta observación podría tener especial interés si consideramos que este oncogén representa una diana terapéutica. En este sentido, estudios experimentales demostraron que anticuerpos monoclonales dirigidos contra c-erbB-2 inhiben el crecimiento de células de cáncer gástrico en cultivo que sobreexpresan el c-erbB- ${ }^{5}$. Asimismo, estudios similares demostraron que la utilización de oligonucleótidos antisentido anti-cerbB-2 inducen incremento de la sensibilidad al cisplatino en el cáncer gástrico ${ }^{6}$.

La mutación del gen p53 es la anomalía genética más común en las neoplasias humanas malignas. Como consecuencia de dicha alteración genética, se produce una proteína anómala, ineficiente para cumplir su función normal de con- trol de la proliferación celular, y con una vida media más prolongada, que ocasiona su fácil acumulación y detección por inmunohistoquímica. Mediante esta técnica se demuestra indirectamente la alteración del gen en más de la mitad de los carcinomas gástricos. Aunque no se ha establecido aun su posible significación pronóstica en esta neoplasia, datos experimentales indican que la p53 puede predecir la respuesta al factor de necrosis tumoral $\alpha$ y al interferón $\gamma$ en líneas celulares derivadas de cáncer gástrico humano ${ }^{7}$. Asimismo, un estudio clínico reciente (fase II) demostró que la tinción inmunohistoquímica positiva para la p53 en las biopsias endoscópicas de cáncer gástrico, es un factor predictivo de respuesta a la quimioterapia neoadyuvante de altas dosis en pacientes con cáncer gástrico avanzado8.

\section{Factores de crecimiento y sus receptores}

Se entiende por regulación autocrina del crecimiento la situación donde una célula tumoral produce un factor estimulante del crecimiento y, poseyendo receptores celulares específicos para él, responde a su estimulación. En los carcinomas gástricos se ha demostrado la sobreexpresión de algunos de esos factores polipeptídicos de crecimiento, tales como el factor de crecimiento tumoral alfa (TGF $\alpha$ ) y el factor de crecimiento epidérmico (EGF) que, a través de su interacción con su receptor de membrana celular (EGFR), estimulan la proliferación de las células tumorales. En el cáncer gástrico, los estudios clínicos describen que la sobreexpresión de este receptor está asociada con una menor supervivencia de los pacientes $^{4,9}$. Además, se ha señalado que la sobreexpresión conjunta del c-erbB-2 y el EGFR identifica un grupo de pacientes con especial mal pronóstico en el cáncer gástrico resecable ${ }^{4}$. Ello parece ser debido a que ambos receptores, pertenecientes a la misma familia de receptores tipo tirosínquinasa, pueden ser activados conjuntamente mediante dimerización. Por otra parte, la acción de ligandos estimuladores sobre el EGFR, a través de diferentes mecanismos tales como inhibidores enzimáticos, anticuerpos u oligonucleótidos antisentido, constituyen una de las principales promesas terapéuticas en oncología. En este sentido, los niveles elevados de expresión tumoral de EGFR podrían tener valor predictivo de cara a seleccionar pacientes con cáncer gástrico candidatos a esas terapias, puestas ya en marcha en ensayos clínicos en fase I-III pare este tipo de neoplasia ${ }^{10}$.

Un aspecto relevante de la progresión de los tumores humanos malignos es el de la promoción de la angiogénesis. En el cáncer gástrico se ha descrito la sobreexpresión de diversos factores estimuladores de este proceso, que fueron encontrados asociados con un peor pronóstico. Tales factores incluyen: factor de crecimiento del endotelio vascular (VEGF), factor de crecimiento hepatocitario (HGF), factor de crecimiento plaquetario (PDGF- $\alpha$ ) y factor transformante del crecimiento betal (TGF- $\beta 1)^{11,12}$. Recientemente también se ha descrito, en un modelo animal experimental, que la combinación de terapia anti-angiogénica y anti-EGFR conduce a una inhibición más efectiva del crecimiento del cáncer gástrico que cada una de esas terapias por separado ${ }^{13}$. Ello sugiere la importancia de combinar dianas terapéuticas de cara a un futuro tratamiento más efectivo.

Otro factor investigado recientemente en la evaluación pronóstica del cáncer gástrico, es la proteína citosólica pS2 
(TFF1). Su expresión en el cáncer gástrico resecable se ha asociado con un peor pronóstico, lo que parece estar posiblemente en relación con el papel del péptido como promotor de la motilidad de las células tumorales ${ }^{14}$.

\section{Moléculas de adhesión y elementos de la matriz extracelular}

La invasión y las metástasis representan un aspecto clave en la progresión de los tumores malignos. Se trata de un proceso muy complejo en el que están involucrados aspectos tan diversos como alteraciones de la motilidad y adhesión celular, alteraciones en la degradación proteolítica de la membrana basal y matriz extracelular. En los carcinomas gástricos se ha descrito una variabilidad de la expresión de diversos parámetros moleculares asociados con esos procesos, lo que resulta indicativo de la heterogeneidad biológica que muestran este tipo de tumores. Así, se ha descrito una expresión reducida de la E-cadherina (molécula de adhesión intercelular calcio-dependiente), que está asociada con un crecimiento tumoral infiltrativo, desarrollo de metástasis y una menor supervivencia de los pacientes ${ }^{15}$. Por el contrario, la sobreexpresión de la subunidad $\alpha 3$ de las integrinas (moléculas de adhesión célula-matriz extracelular) ${ }^{16}$, o de CD44 (molécula de adhesión glicosilada que está involucrada en la adhesión intercelular y entre la célula y matriz extracelular) ${ }^{17}$, se han relacionado con un peor pronóstico. Asimismo, la alteración de determinados elementos de la matriz extracelular se ha demostrado que tienen relevancia pronóstica. En este sentido, recientemente hemos descrito que las concentraciones intratumorales elevadas de ácido hialurónico están asociadas con un peor pronóstico en el cáncer gástrico resecable ${ }^{18}$. Esto puede ser debido a que los elevados niveles tisulares del ácido hialurónico pueden ocasionar separación de las barreras de colágeno, facilitando así la migración de las células tumorales y la invasión tisular. Además, las células tumorales recubiertas de ácido hialurónico pueden protegerse del ataque de linfocitos citotóxicos y, probablemente, de agentes quimioterápicos.

\section{Enzimas proteolíticos y sus inhibidores}

Hoy es sabido que las células tumorales utilizan enzimas proteolíticos para penetrar en el tejido circundante y desencadenar metástasis. Entre las proteasas cuya expresión elevada ha sido asociada con un pronóstico desfavorable en el cáncer gástrico, se encuentra la cisteín-proteasa catepsina $B$, las aspartil-proteasas catepsina $D$ y $E$, las serín-proteasas uPA y IPA, y las metaloproteasas gelatinasa A, matrilisina, metaloproteasa de membrana tipo 1 y colagenasa- $3^{19-22}$. No obstante, el papel de las proteasas en la biología del cáncer es más complejo de lo que se pensaba en un principio. Así, existen otras proteasas, tales como la aspartil-proteasa pepsinógeno $C$, cuya expresión está asociada con un pronóstico favorable en el cáncer gástrico ${ }^{23}$. Ello puede ser debido a que en este último caso la expresión de la proteasa, normalmente producida por toda la mucosa gástrica, parece estar más bien en relación con una buena diferenciación funcional de los tumores de esta localización. Aún así, la inhibición se- lectiva de las proteasas especialmente involucradas en la progresión del cáncer gástrico, podría representar una nueva estrategia terapéutica en este tipo de neoplasia. De hecho, existen ya estudios preliminares en el cáncer gástrico avanzado que muestran actividad anticancerosa del marimastato: un inhibidor sintético de las metaloproteasas ${ }^{24}$

\section{Conclusiones}

A pesar de lograr una resecabilidad quirúrgica aparentemente curativa del cáncer gástrico, este tipo de tumor sigue mostrando una elevada tasa de recurrencias tumorales que, en la práctica mayoría de los casos, ocasionan mortalidad. De ahí la importancia de una más correcta evaluación pronóstica en este tipo de neoplasia, de cara a una adecuada selección de pacientes candidatos a tratamientos más agresivos. La consideración de los factores pronósticos derivados de la biología molecular, puede complementar la información aportada por los factores pronósticos clásicos del cáncer gástrico. Además, esos nuevos factores pueden tener valor predectivo de la respuesta a agentes quimioterapéuticos clásicos o a futuras estrategias basadas en nuevas dianas terapéuticas. No obstante, resulta necesario, en la actualidad, integrar toda esa nueva información aportada por los parámetros de la biología molecular de los carcinomas gástricos, con el objeto de identificar los diferentes fenotipos del comportamiento tumoral de este tipo de neoplasia, con sus posibles implicaciones terapéuticas.

\section{Bibliografía}

1. Kelley JR, Duggan JM. Gastric cancer epidemiology and risk factors. J Clin Epidemiol 2003; 56:1-9.

2. Danesi DT, Spano M, Fabiano A, Altavista P, Pasqualetti $P$, Toscano MG, Antonini F, Catalano P, Mecozzi A, Picconi A, Daffina A, Cucchiara G. Flow cyłometric DNA ploidy, p53, PCNA, and c-erbB-2 protein expressions as predictors of survival in surgically resected gastric cancer patients. Cytometry 2000; 42:27-34

3. García I, del Casar JM, Corte MD, Allende MT, García-Muñiz $\mathrm{JL}$, Vizoso F. Epidermal growth factor receptor and c-erbB-2 contents in unresectable (UICC R1 or R2) gastric cancer. Int J Biol Markers 2003; 18:200-6.

4. García I, Vizoso F, Martín A, Sanz L, Abdel-Lah O, Raigoso P, García-Muñiz JL. Clinical significance of the epidermal growth factor receptor and HER2 receptor in resectable gastric cancer. Ann Surg Oncol 2003; 10: 234-41.

5. Kono K, Takahashi A, Ichihara F, Sugai H, Fujii H, Matsumoto Y. Impaired antibody-dependent cellular cytotoxicity mediated by herceptin in patients with gastric cancer. Cancer Res 2002; 62:5813-7.

6. Funato T, Kozawa K, Fujimaki S, Miura T, Kaku M. Increased sensitivity to cisplatin in gastric cancer by antisense inhibition of the her-2/neu (c-erbB-2) gene. Chemotherapy 2001; 47:297303.

7. Fukui T, Matsui K, Kato H, Takao H, Sugiyama Y, Kunieda K, Saji S. Significance of apoptosis induced by tumor necrosis factor-alpha and/or interferon-gamma against human gastric cancer cell lines and the role of the p53 gene. Surg Today 2003; 33:847-53. 


\section{F. J. Vizoso Piñeiro y cols.}

8. Bataille F, Rummele P, Dietmaier W, Gaag D, Klebl F, Reichle A, Wild P, Hofstadter F, Hartmann A. Alterations in p53 predict response to preoperative high dose chemotherapy in patients with gastric cancer. Mol Pathol 2003; 56:286-92.

9. D'Agnano I, D'Angelo C, Savarese A, Carlini M, Garofalo A, Bottari L, Santoro E, Giannarelli D, Vecchione A, Zupi G. DNA ploidy, proliferative index, and epidermal growth factor receptor: expression and prognosis in patients with gastric cancers. Lab Invest 1995; 72:432-8.

10. Kopp R, Rothbauer E, Ruge M, Arnholdt H, Spranger J, Muders $M$, Pfeiffer DG, Schildberg FW, Pfeiffer A. Clinical implications of the EGF receptor/ligand system for tumor progression and survival in gastrointestinal carcinomas: evidence for new therapeutic options. Recent Results Cancer Res 2003; 162:115-32.

11. Saito $H$, Tsujitani $S$, Oka $S$, Kondo A, Ikeguchi $M$, Maeta $M$, Kaibara $\mathrm{N}$. The expression of transforming growth factor-betal is significantly correlated with the expression of vascular endothelial growth factor and poor prognosis of patients with advanced gastric carcinoma. Cancer 1999; 86:1455-62.

12. Shi $H, X u J M, H u N Z$, Xie HJ. Prognostic significance of expression of cyclooxygenase- 2 and vascular endothelial growth factor in human gastric carcinoma. World J Gastroenterol 2003; 9:1421-26.

13. Jung YD, Mansfield PF, Akagi M, Takeda A, Liu W, Bucana $C D$, Hicklin DJ, Ellis LM. Effects of combination anti-vascular endothelial growth factor receptor and anti-epidermal growth factor receptor therapies on the growth of gastric cancer in a nude mouse model. Eur J Cancer 2002; 38:1133-40.

14. Suárez C, Vizoso F, Rodríguez JC, García I, Raigoso P, Allende MT, García-Muñiz JL, García-Morán M. Prognostic significance of cytosolic pS2 protein content in gastric cancer. Int J Biol Markers 2001; 16:37-44

15. Zhou YN, Xu CP, Han B, Li M, Qiao L, Fang DC, Yang JM. Expression of $\mathrm{E}$-cadherin and beta-catenin in gastric carcinoma and its correlation with the clinicopathological features and patient survival. World J Gastroenterol 2002; 8:987-93.
16. Boku N, Yoshida S, Ohtsu A, Fujii T, Koba I, Oda Y, Ryu M, Matsumoto T, Hasebe T, Hosokawa K, et al. Expression of integrin alpha 3 in gastric and colorectal cancers: its relation to wall contraction and mode of invasion. Jpn J Cancer Res 1995; 86:934-40.

17. Muller W, Schneiders A, Heider KH, Meier S, Hommel G, Gabbert HE. Expression and prognostic value of the CD44 splicing variants v5 and v6 in gastric cancer. J Pathol 1997; 183:2227.

18. Vizoso F, del Casar JM, Corte MD, García I, Corte MG, Alvarez A, García-Muñiz JL. Significance of cyłosolyc hyaluronan levels in gastric cancer. Eur J Surg Oncol in press.

19. Sanz L, Vizoso F, Verez P, Allende MT, Corte MG, Abdel-Lah O, Martín A, García-Muñiz JL. Prognostic significance of tissuetype plasminogen activator (tPA) content in gastric cancer and surrounding mucosa. Int J Biol Markers 2002; 17:169-76.

20. del Casar JM, Vizoso F, González LO, Martín A, Gava R, Cuesta $E$, Diez MC. Expression and clinical significance of collagenase-3 (MMP-13) in gastric cancer. Gastroenterol Hepatol 2003; 26:1-7.

21. Kaneko T, Konno H, Baba M, Tanaka T, Nakamura S. Urokinase-type plasminogen activator expression correlates with tumor angiogenesis and poor outcome in gastric cancer. Cancer Sci 2003; 94:43-9

22. Vizoso F, Martínez A, Vázquez J, Lamelas M, Rodil A, GarcíaMuñiz J. Significación biológica y clínica de los enzimas proteolíticos y sus inhibidores en los carcinomas humanos. Cirugía Española 2000; 68:471-85.

23. Fernández R, Vizoso F, Rodríguez JC, Merino AM, González LO, Quintela I, Andicoechea A, Truan N, Diez MC. Expression and prognostic significance of pepsinogen $C$ in gastric carcinoma. Ann Surg Oncol 2000; 7: 508-14.

24. Tierney GM, Griffin NR, Stuart RC, Kasem H, Lynch KP, Lury JT, Brown PD, Millar AW, Steele RJ, Parsons SL. A pilot study of the safety and effects of the matrix metalloproteinase inhibitor marimastat in gastric cancer. Eur J Cancer 1999; 35:563-8. 